\title{
All-Optical Multicasting on Wavelength-Routed WDM Networks with Partial Replication ${ }^{\dagger}$
}

\author{
Wen-Yu Tseng and Sy-Yen Kuo \\ Department of Electrical Engineering, National Taiwan University, Taipei, Taiwan \\ Email: sykuo@cc.ee.ntu.edu.tw
}

\begin{abstract}
An All-Optical Multicast Heuristic (AOMH) method is proposed to perform all-optical multicasting on WDM networks. AOMH first divides the members of a multicast group into MCG (multicast-capable group) and MIG (multicast-incapable group). If MCG is empty or the fanout constraints are not satisfied, AOMH will add some Proxy MC (PMC) nodes in MCG to satisfy the constraints Then the multicast tree is determined in three steps. First AOMH constructs a multicast tree for MCG. Second, AOMH partitions MIG into several sub-MIGs according to the distances to the members of MCG. Finally, AOMH generates the multicast sub-trees rooted at the members of MCG with the destinations of their own sub-MIGs. AOMH has good performance if the number of MC nodes is larger than $20 \%$ of the number of nodes in a network. Furthermore, if a node has a higher degree, multicast capable devices will be required to improve the network performance.
\end{abstract}

\section{Introduction}

Wavelength Division Multiplexing (WDM) networks are emerging as the major technology to divide the enormous capacity of a single-mode fiber into concurrent channels [1]. Accompanied with the advent of bandwidth-driven applications, WDM networks for the Next Generation Internet (NGI) need to provide multicast services with high throughput and small latency [2]. WDM multicast can be achieved using the existing conventional multicasting approaches over the IP layer; i.e., construction of a multicast tree with DVMRP, CBT, OSPF, DDMC or PIM [3-6]. However, in this way we cannot achieve All-Optical Multicasting (AOM) on WDM networks over the optical layer because IP multicasting has to duplicate packets in the routers electronically. In other words, this approach sacrifices the benefits of all-optical routing and results in replication and processing latencies of $\mathrm{O} / \mathrm{E} / \mathrm{O}$ (opti$\mathrm{cal} /$ electrical/optical) conversions.

AOM, same as IP multicast, constructs a distribution tree with specified Quality of Service (QoS) such as end-to-end delay and overall bandwidth. This is similar to the standard Steiner tree problem and there are many existing heuristics with different objectives. With a distribution tree

\footnotetext{
${ }^{\dagger}$ Acknowledgment: This research was supported by the National Science Council, Taiwan, R. O. C., under the Grant NSC 89-2213-E002-114.
}

determined by these heuristics, multicasting can potentially share many links transmitting packets from the source node to the destination nodes. In addition, it also minimizes packet replication in the source node because the fork nodes in the tree help to replicate packets. If the nodes do not support multicasting, either the distribution tree or the protocols have to be modified to bypass such nodes. For IP multicasting, construction of the tree can simply ignore these nodes as in MOSPF [4], or uses IP-in-IP encapsulation/tunneling as in DVMRP [5] such that multicasting can be treated as unicast traffic. For AOM, however, these nodes cannot just be ignored and the packets cannot be processed optically as unicast traffic.

Because AOM has to distribute packets in optical domain, whether the architecture of an optical switch can support multicasting is an important issue. Each node should be equipped with a Multicast-capable Wavelength Routing Switch (MWRS) to support multicasting, which performs better than Linear Divider-Combiner (LDC) $[7,12,14]$. An optical switch with the multicasting capability is called a Multicast Capable (MC) node, and otherwise called a Multicast Incapable (MI) node [7]. Different from the definition of a switch in MBONE [11], an MI node cannot be spanned because optical switches cannot support encapsulation/tunneling. Thus in a multicast distribution tree, MI nodes must connect to the source node or the MC nodes with unicast paths. If an MI node is not a destination node, it can be used in a distribution tree as an intermediate node to forward the multicast packets to a sub-tree of downstream destinations. If it is a source or a destination node, it always connects to the tree through a single branch. Therefore, AOM is inherently different from IP multicast on managing MI nodes.

In this paper, we propose an All-Optical Multicast Heuristics (AOMH) for WDM networks with Partial Packet Replication (PPR). AOMH first partitions members of the multicast group into the MC Group (MCG) and the MI Group (MIG). If MCG is empty or the fanout constraints are not satisfied, AOMH will select some Proxy MC (PMC) nodes to include in the MCG to satisfy the constraints. Then the multicast tree is determined in three steps. First, AOMH constructs a multicast tree for MCG with the shortest-path heuristics, delay-constrained heuristics or the shared-tree heuristics. Second, AOMH partitions the MIG into several sub-MIGs according to the distances to members of MCG Finally, AOMH constructs multicast sub-trees rooted at members of MCG with destinations of their own sub-MIGs. In fact, the sub-trees in the third step are similar to CBT because each MI destination is directly connected to the nearest member in MCG 
From the AOMH, the determined multicast tree is a real all-optical multicast tree to transmit multicast traffic on a WDM network. However, the number of MC nodes in the network affects AOMH significantly. The simulation results show that if the number of MC nodes is less than $20 \%$ of the number of nodes in a network, the number of wavelengths reserved for links near MC nodes increase dramatically because most of the multicast trees connect to the $\mathrm{MC}$ nodes. In addition, the maximum number of wavelengths for AOMH that needs be supported by the state-of-the-art commercial products, even with the number of wavelength suggested in [8], is too large. Besides, the number of reserved links and the delay from source to destinations for a multicast request will also increase even if the traffic load is small. In other words, AOMH results in good performance for a network with the number of $\mathrm{MC}$ nodes larger than $20 \%$ of the total number of nodes. Furthermore, the MC node deployment strategies also affect $\mathrm{AOMH}$. The first strategy, called RAND, is to just deploy the MC nodes randomly. The second strategy, called PRIOR, is to deploy the MC nodes semi-randomly according to the node degree i.e., the node with more output links has a higher priority to be an MC node. The results also show that PRIOR has better performance than RAND. Therefore, AOMH provides a good all-optical multicast solution in WDM networks as long as the number of MC nodes is enough and the MC nodes deploy well.

The rest of this paper is organized as follows. The all-optical multicasting problem is defined in Section 2 . Three multicast capable node deployment strategies are also introduced in this section. In Section 3, we propose the All-Optical Multicast Heuristics with examples. Performance metrics are defined and simulation results are presented in Section 4. The contributions of this paper are summarized in Section 5.

\section{Preliminaries}

\subsection{Network and Multicasting Model}

A WDM network is modeled by a simple and connected graph $G(V, E, \Lambda)$ with degree $\delta$, where a node $v, v \in V,|V|=$ $N$, represents a network node and a link $e, e \in E$, corresponds to a communication link between two nodes. We assume that $\Lambda=\left\{\lambda_{1}, \ldots, \lambda_{k}\right\}$ of wavelengths in the network. For each link $e$ from node $g$ to node $h$ and wavelength $\lambda_{i}$, a cost $c_{t}\left(g, h: \lambda_{i}\right), g \neq h$, is given to represent the cost of using wavelength $\lambda_{i}$ to transmit from node $g$ to node $h$. Besides, $c_{i}\left(g, h: \lambda_{i}\right)=\infty$ if $g=h$. If $\lambda_{i}$ is not available from node $g$ to node $h, c_{t}\left(g, h: \lambda_{i}\right)=\infty$ (or a very large number for practical number representation). The cost of wavelength conversion at node $i$ from wavelength $\lambda_{p}$ to $\lambda_{q}$ is modeled by $c_{c}\left(i: \lambda_{p}, \lambda_{q}\right)$. For some nodes with sparse conversion, where the wavelength conversion from $\lambda_{p}$ to $\lambda_{q}$ is not available, $c_{c}\left(i: \lambda_{p}, \lambda_{q}\right)=\infty$ (or a very large number). If the two wavelengths are the same, the cost is zero; that is, $c_{c}\left(i: \lambda_{p}, \lambda_{p}\right)=0$. For multicast transmission, the cost of light splitting $c_{s}(w)$ for node $w, w \in V$, has a certain value if node $w$ is an MC node. $c_{s}(w)$ depends on the maximum number of the light splitters and the number of destinations that node $w$ wants to connect in a multicast session. If node $w$ is an MI node, $c_{s}(w)=\infty$. In addition, it is obvious that $c_{t}, c_{c}$, and $c_{s}$ are all nonnegative.
The above-defined cost of wavelength conversion accommodates the general case where conversion costs depend on the number of nodes and wavelengths involved, a case often encountered in practical networks. For example, wavelength convertible nodes in critical locations in a network (e.g. nodes used to interconnect different networks) should be used only when necessary and therefore should be assigned higher conversion costs. When wavelengths are grouped into wavebands, conversion between wavelengths in the same waveband is generally less costly than conversion between wavelengths in different wavebands. The conversion costs should be assigned. For example, the costs can be easily assigned in such a way that the least numbered available wavelengths will be used first. Furthermore, conversion costs can be dynamically adjusted to adapt to changing network conditions. For example, when the traffic going through a wavelength conversion node is approaching the node's capacity, wavelength conversion cost at this node can be increased so that new transmission paths can be routed through other nodes.

The multicast transmission in $G(V, E, \Lambda)$ involves a multicast group and a multicast tree. The multicast group $D$, $D \subseteq V$, consists of a source node $s$ and several destination nodes; that is, $|D|=2$ for unicast, $2<|D|<|\eta|$ for multicast, and $|D|=\mid \eta$ for broadcast. The multicast tree $T\left(V_{T}, E_{T}, \Lambda_{T}\right)$ rooted on $s$ transmits multicast packets to the destination nodes, where $T \subset G, D \subset V_{T} \subset V, E_{T} \subset E$ and $\Lambda_{T} \subset \Lambda$. The tree cost $C(T)$ of $T$ is represented as

$$
\begin{aligned}
C(T)= & \sum_{f, g \in V_{T}, \lambda_{i} \in \Lambda_{T}} c_{t}\left(f, g: \lambda_{i}\right) \\
& +\sum_{h \in V_{T}, \lambda_{j}, \lambda_{k} \in \Lambda_{T}} c_{c}\left(h: \lambda_{j}, \lambda_{k}\right)+\sum_{h \in V_{T}} c_{s}(w),
\end{aligned}
$$

which is the optimization objective for multicast routing. This can be formulated as the All-Optical Steiner Tree problem (AOST). Because the AOST problem can be easily reduced to the standard Steiner Tree problem, the AOST problem is NP-complete. Thus heuristics are needed to solve AOST.

\subsection{Node Deployment}

Based on the MC node management of AOM, different MC node deployment strategies affect the performance of AOM. The first is Full Packet Replication (FPR), where all nodes are MC nodes. This is a trivial case of $\mathrm{MC}$ node deployment because heuristics of IP multicast can directly construct a tree to achieve all-optical multicast. Besides, all-optical multicasting with FPR will result in good performance in terms of delay and the number of wavelengths. However, FPR may not exist in a real network because it is too expensive to have light splitters in every node. The second is No Packet Replication (NPR), where all nodes are MI nodes. With this deployment, the multicast mechanism cannot be directly applied to construct a distribution tree. The source node must first replicate the multicast packets and reserve the unicast paths to destination nodes. The copies of the packets are transmitted through unicast paths. Obviously the number of destinations $D, D<\delta W$, limits the number of unicast paths reserved by the source node where $\delta$ is the degree of the source node and $W$ is the number of wavelengths. If the above constraint is not satisfied, AOM cannot be achieved. Besides, research in [9] reported that bandwidth utilization of NPR is unacceptable. 
The third MC deployment strategy is Partial Packet Replication (PPR), where some nodes are MC nodes and others are MI nodes. This is a more practical and challenging case for AOM. The research proposed in [10] provides four heuristics of WDM multicast, with the assumption that every switch can support "drop and continue" operation, even in MI nodes. This operation in MI nodes uses add-drop multiplexer to receive packets through $\mathrm{O} / \mathrm{E}$ and forward copies through E/O. Thus these heuristics are not for AOM. In fact, MI nodes can only support "drop only" and "continue only" operations for all-optical transmission. In addition, they will be always leaf nodes connecting to MC destinations through unicast paths in a tree if they are destinations. The number of unicast paths reserved for MI destinations will be limited by the fanout constraint $f=W \sum \delta_{i}>D$, where $\delta_{i}$ is the degree of the $i$ th MC destination, and the number of unicast paths through a specific link connected to a MC destination should be smaller than $W$. Besides, the way MC nodes are deployed will also influence the performance of AOM.

\section{All-Optical Multicast}

\subsection{MWLG Models for AOM}

In order to show how the all-optical multicasting heuristic is working on the WDM network, the graph of $G(V, E, \Lambda)$ has to be converted to a Modified Wavelength-Layered Graph (MWLG) model. Similar to the model defined in $[12,13]$, let us first consider the wavelength-routed optical network shown in Figure 1(a), which can be modeled as a layered graph shown in Figure 1(b). Each layer in the graph represents a wavelength except $\lambda_{0}$, and each physical fiber link has a corresponding link on each wavelength layer. Different from the definition of $\lambda_{0}$ as a control wavelength in $[12,13]$, here $\lambda_{0}$ represents the electronic interface of each wavelength for each node. Thus there always exists a link for node $n$ connecting from $\left(n, \lambda_{0}\right)$ to $\left(n, \lambda_{i}\right), i=1,2, \ldots$. According to the regular WLG model in $[12,13]$, a control wavelength can be represented by one of $\lambda_{i}, i=1,2, \ldots$ instead and does not lose the generality. A link for node $n$ from $\left(n, \lambda_{i}\right)$ to $\left(n, \lambda_{j}\right), i, j \neq 0, i \neq j$, represents wavelength conversion from $\lambda_{i}$ to $\lambda_{j}$ on node $n$. Figure 1(b) represents a full wavelength conversion WDM network and the fully connected graph for wavelength conversion is reduced to a bold vertical line for simplicity. In fact a sparse wavelength conversion WDM network can be also represented as long as some links of the wavelength conversion graph are disconnected.

Based on the MWLG model, we can demonstrate all-optical multicasting on a WDM network with the three MC node deployments. Assume that there exists a multicast transmission from node 1 , with the destination nodes 2 , 3,4 , and 6 on the network shown in Figure 1(a). First, consider the NPR deployment. According to the minimization objective defined in Eq. (1), the cost of light splitting is infinite for the NPR deployment. Thus the Steiner heuristics can not be used. The only way to support multicasting is to duplicate the packets in the electronic switch of the

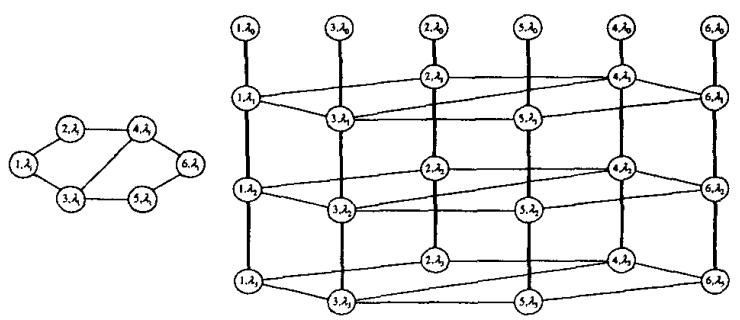

(a)

(b)

Figure 1. Modified Wavelength-Layered Graph: (a) original network, (b) layered graph.

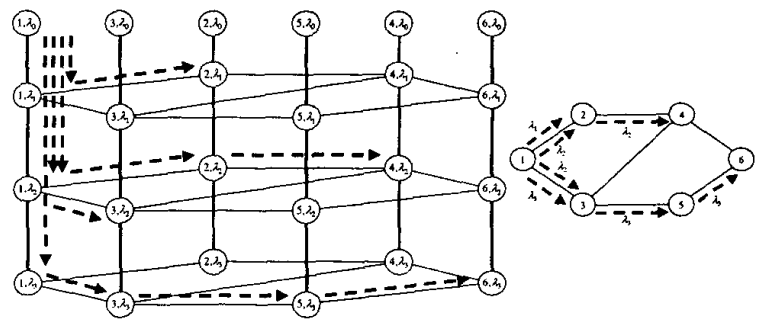

(a)

(b)

Figure 2. AOM for NPR: (a) distribution tree on MWLG, (b) wavelength assignment.

source node and to reserve unicast lightpaths from source to each destination. As shown in Figure 2, because there is no MC node in the network, node 1 has to request unicast lightpaths to node $2,3,4$, and 6 , respectively. Packets of the multicast transmission have to be duplicated as 4 copies on node 1 , and sent to node $2,3,4$, and 6 along their specific lightpaths. (The arrows back to $\lambda_{0}$ of node $2,3,4$, and 6 are neglected for simplicity.) The distribution tree is similar to the FAT-tree [15] and the workload on node 1 is 4 times larger than that of unicast transmission. Besides, the number of wavelengths reserved from node 1 is 4 , which is equal to the number of destinations. That is, the number of destinations is limited by the number of unicast lightpaths that the server node can reserve at the same time. Therefore, AOM with NPR will keep the server node busier in the multicast transmission than in the unicast transmission.

Next, consider the FPR deployment. Because all nodes are MC nodes in the network, the cost of light splitting $c_{s}$ for each node can be neglected for simplicity. Thus the objective in Eq. (1) is reduced to the standard Steiner objective and the Steiner tree heuristics can be directly used to construct a multicast tree on the modified wavelength-layered graph. As shown in Figure 3, the multicast tree with wavelength assignment can be easily determined on MWLG. Note that the multicast packets are not duplicated on the electronic switch of node 1 . When the packets arrive at the fork node of the tree (node 4), they are duplicated by the light splitter, not by the electronic switch. Thus the workload on node 1 is the same as that of unicast transmission and the number of wavelengths assigned from node 1 is 1 . Therefore, AOM with FPR is an ideal case that supports multicasting in nature.

Finally, consider the PPR deployment. Because some nodes are MC nodes and others are MI nodes, the cost of light splitting cannot be neglected. Thus the Steiner tree heuristics cannot be directly used. We have to partition the 


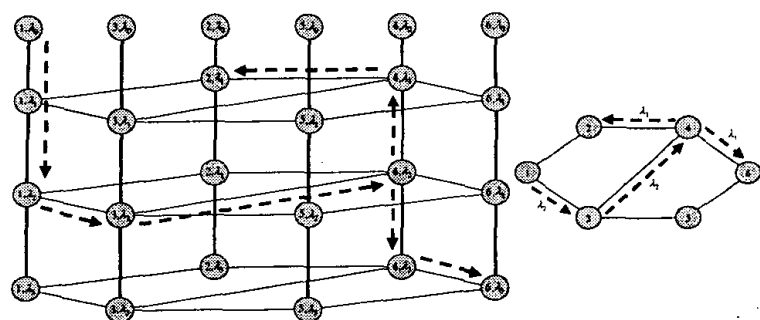

(a)

(b)

Figure 3. AOM for FPR: (a) distribution tree on MWLG, (b) wavelength assignment.

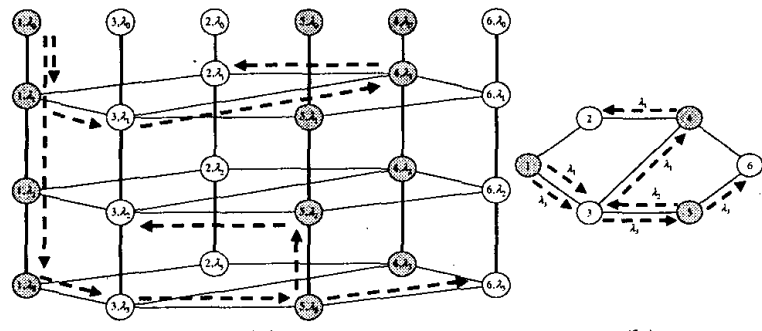

(a)

(b)

Figure 4. AOM for PPR: (a) distribution tree on MWLG, (b) wavelength assignment.

multicast group into sub-groups. First the nodes in the multicast group that are MC nodes are selected and construct a distribution sub-tree, called an MCG tree. If a fork node in the MCG tree is an MI node, the packet duplication is executed in the parent MC node, which remains in the optical domain. Then the MI nodes in the multicast group connect to the nearest $\mathrm{MC}$ nodes of the multicast group via the unicast transmission paths. In other words, each MC node and its connected MI nodes of the multicast group construct a sub-tree called an MIG tree. If the fanout constraint in MCG tree is too small to support the MIG tree connections, an additional $\mathrm{MC}$ node has to be selected as Proxy MC node, or PMC node. As shown in Figure 4, node 1, 4, and 5 are MC nodes, and node 2, 3, and 6 are MI nodes. Only node 1 and 4 are MC nodes in the multicast group and we assume that the fanout constraint is too small. Thus node 5 is selected as the PMC node and the MCG tree is constructed by including node 1,4 , and 5 . The fork node of the MCG tree is node 3 , which is an MI node. Thus packet replication is done in node 1 , and two paths from node 1 flow through node 3 . Then node 2 connects to node 4 , and node 3 and 6 connect to node 5 . These two trees are MIG trees.

According to the tree construction mentioned above, the distribution tree may be criticized at a glance that it does not follow the rule of the Steiner tree. As shown in Figure 4(b), two branches are reserved between node 3 and node 5 to be a cycle. In fact, as shown in Figure 4(a), the tree construction mechanism is cycle-free because the branch from node 3 to node 5 uses $\lambda_{3}$ in the MCG tree, and the branch from node 5 to node 3 uses $\lambda_{2}$ in the MIG tree. Besides, the MCG tree contains two distinct routing paths through the optical switch of node 3 and the packets are duplicated on the light splitter in node 1 . Thus the workload on node 1 is also the same as that of unicast transmission and the number of wavelengths assigned by node 1 is 1 . Moreover, if the source node (node 1 ) is the MI node, it still transmits the packets to the nearest MC node (node 4 for instance) in the MCG tree; and the constructed tree is still cycle-free. Based on the MWLG models discussed above, multicasting on NPR constructs an MIG tree and on FPR an MCG tree. Thus AOMH treats FPR as a special case of PPR, not NPR. Therefore, all-optical multicasting can be achieved in a WDM network.

\subsection{AOM Heuristic}

According to the context described above, an all-optical multicasting heuristic (AOMH) can be deduced for FPR and PPR. AOMH is described as follows.

$\operatorname{AOMH}(S, D, G(V, E, \Lambda))$

$\mathrm{MG}=\{S, D\}, S$ is the source node and $D$ are destinations

$G(V, E, \Lambda)$ is a WDM network

1. Partition MG into MCG and MIG

2. Calculate fanout constraint $f_{M C O}$

3. while $f_{M C G}<|D|$

4. Select a Proxy MC node and add to MCG

5. Calculate $f_{M C G}$

6. Construct an MCG tree in $G(V, E, \Lambda)$

7. for each member $n_{i}$ in MIG

8. Find a nearest member $n_{c}$ in $\mathrm{MCG}$

9. if $n_{i}=S$

10. Construct a unicast lightpath from $n_{i}$ to $n_{c}$

11. else

12. Construct a unicast lightpath from $n_{c}$ to $n_{i}$

Note that the fanout constraint $f_{M C G}=W \sum \delta_{i}$, while $\delta_{i}$ is the degree of the $i^{\prime}$ th node in MCG Define that the splitting ratio is the ratio of the number of $\mathrm{MC}$ nodes to the number of all network nodes. The selection of the Proxy MC node in step 3-5 will execute frequently when the splitting ratio is very small. In step 6 , the Steiner tree heuristics can be used, such SPT, DDMC, or delay-constrained heuristics. In addition, the size of MCG increases as the splitting ratio increases. In step 9-12, the construction of a unicast lightpath can be determined by the existing algorithms for unicast transmission. In addition, the size of MIG may decrease as the splitting ratio increases. The complexity of AOMH depends mainly on MCG tree construction with fewer multicast destinations. Therefore, AOMH is an efficient heuristics for all-optical multicasting on a WDM network with the construction of conventional Steiner tree and unicast lightpaths.

\section{Simulation Results}

As described in the previous section, the splitting ratio is an important factor for $\mathrm{AOMH}$. In this section, we present a simulation-based study to depict the performance of AOMH for the networks with different splitting ratios. We simulated AOMH on randomly generated, sparse, 50-node networks with network degree $\delta \cong 3$. In order to be close to a real network, the generated networks were assembled in a manner similar to those used in $[16,17]$. Each of the 50 vertices is distributed across a Cartesian coordinate plane 
with minimum coordinate $=(0,0)$ and maximum coordinate $=(100,100)$ which creates a forest of 50 vertices spread across the plane. Then these vertices are connected with a random spanning tree. The remaining redundant edges of the network are chosen by examining each possible edge $(x, y)$ and generating a random number $0 \leq q<1$. Edges are introduced with a probability that depends on the distance of $(x, y)$. The edge probability is given by

$$
\dot{P}(x, y)=\beta \cdot \exp \left(\frac{-d(x, y)}{100 \alpha}\right) \text {. }
$$

If $q<P(x, y)$, this edge is accepted. The parameters $\alpha$ and $\beta$ characterize the network graph. The density of links can be increased with increasing $\beta$; in addition, the link density of short links relative to that of longer ones can be reduced with increasing $\alpha$. We choose $\alpha=0.075$ and $\beta=$ 0.075 for generating the graphs with $\delta=3$; the relationship among $\alpha, \beta$, and $\delta$ is thoroughly discussed in [17]. Based on the definition of simple and connected networks, nodes with only one incident edge were avoided.

The performance metrics to evaluate the efficiency of the heuristics include the number of reserved links, the average delay, and the average and maximum number of reserved wavelengths for $D=5$ and $D=10$. The packet generation rate is 0.2 and the service rate is 1 . The destinations are randomly chosen. The heuristics of MCG tree constructions are SPT and DDMC. The heuristics of MIG constructions are SPT because unicast transmission usually needs a shortest path connection. Two strategies of MC node deployment are also simulated for comparison. The first strategy, called RAND, is to just deploy the MC nodes randomly. The second strategy, called PRIOR, is to deploy the MC nodes semi-randomly according to the node degree; that is, the node with more output links has a higher priority to be an MC node. The metrics for NPR are also depicted for comparison. The $95 \%$ confidence interval of each data point is calculated.

Figure 5 shows the average number of reserved links for AOMH. The number of reserved links for the MCG tree is $L_{m c}$, the number of reserved links for the MIG tree is $L_{c i}$, and the number of total reserved links is $L_{p}$. Note that $L_{m c}$ increases and $L_{c i}$ decrease as the splitting ratio increases. $L_{m c}$ and $L_{c i}$ are almost the same when the splitting ratio is about 0.55 . $L_{p}$ reaches a constant value with SPT and still decreases with DDMC as the splitting ratio increases. $L_{p}$ for NPR is highest no matter what the splitting ratio is. Besides, PRIOR reserves fewer links than RAND.

Figure 6 shows the average root-to-leaf delay for AOMH. The average root-to-leaf delay for the MCG tree is $D_{m c}$, the average root-to-leaf delay for the MIG tree is $D_{c i}$, and the average source-to-destination delay is $D_{p}$. Note that $D_{m c}$ increases and $D_{c i}$ decrease as the splitting ratio increases. $D_{m c}$ and $D_{c i}$ are almost the same when the splitting ratio is about 0.4 for $D=5$ and 0.2 for $D=10$. $D_{p}$ for AOMH is approximately $25 \%$ larger than that for NPR and smaller when the splitting ratio is larger than 0.8 with SPT. Thus the delay for AOMH is acceptable for multicasting. Besides, PRIOR results in smaller delay than RAND.

Figure 7 shows the average number of reserved wavelengths per multicast session for AOMH. The average number of reserved wavelengths for the MCG tree is $W_{m c}$, the average number of reserved wavelengths for the MIG tree is $W_{c i}$, and the average reserved wavelengths for the AOMH tree is $W_{t}$. PRIOR also reserves fewer wave-

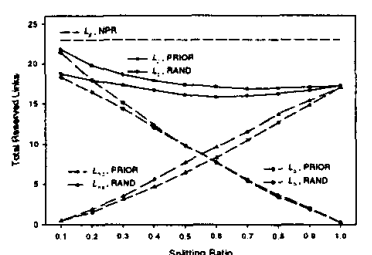

(a)

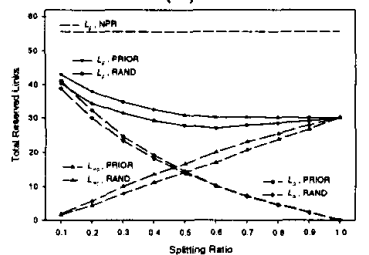

(c)

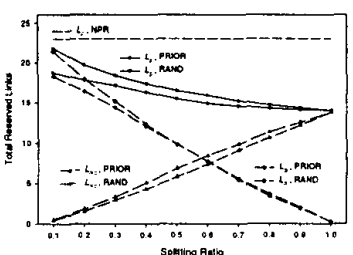

(b)

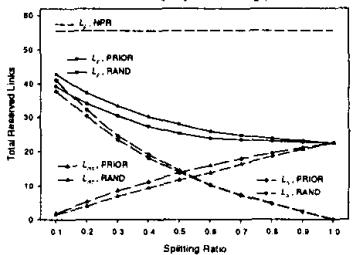

(d)
Figure 5. Average number of reserved links : (a) $D=5$ for SPT, (b) $D=5$ for DDMC, (c) $D=10$ for SPT, and (d) $D$ $=10$ for $\mathrm{DDMC}$.

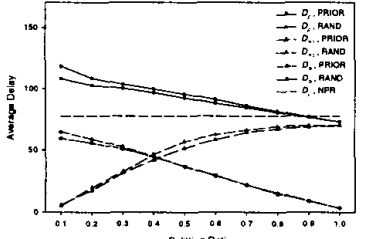

(a)

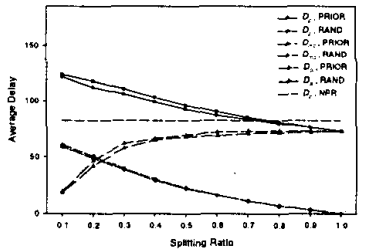

(c)

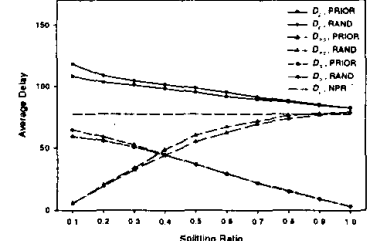

(b)

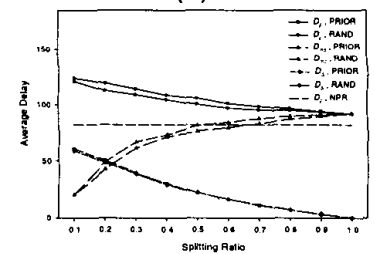

(d)
Figure 6. Average root-to-leat delay: (a) $D=5$ for SPT, (b) $D=5$ for DDMC, (c) $D=10$ for SPT, and (d) $D=10$ for DDMC.

lengths than RAND, but not very significant. Besides, DDMC will reserve fewer wavelengths than SPT as the splitting ratio increases.

Figure 8 shows the maximum number of reserved wavelengths in a link for AOMH. PRIOR reserves fewer wavelengths than RAND. DDMC will reserve fewer wavelengths in a link than SPT as the splitting ratio increases. - Besides, AOMH reserves fewer wavelengths than NPR when the splitting ratio is larger than 0.2 . If the splitting ratio is smaller than 0.2 , the number of reserved wavelengths for $A O M H$ will increase dramatically because the MC nodes will be request in most multicast transmissions. The maximum number of wavelengths will occur in the link near the MC node, and the workload of the MC node will be very high. When the splitting ratio is 0.5 , the maximum number of reserved wavelengths is 10 for $D=5$ and 15 for $D=10$. This can be easily supported by the state-of-the-art WDM technology.

According to the simulation results depicted above, all metrics are better than those for NPR except the 


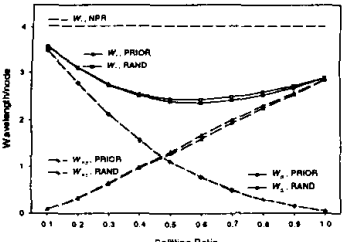

(a)

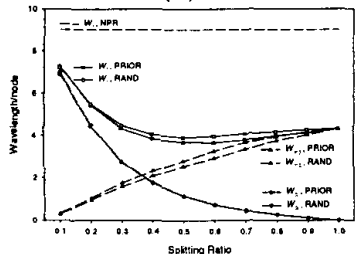

(c)

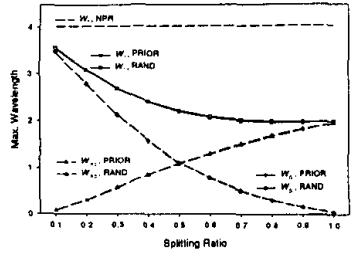

(b)

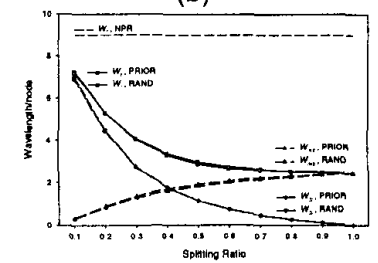

(d)
Figure 7. Average number of reserved wavelengths per session : (a) $D=5$ for SPT, (b) $D=5$ for DDMC, (c) $D=$ 10 for SPT, and (d) $D=10$ for DDMC.

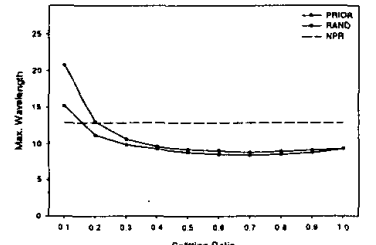

(a)

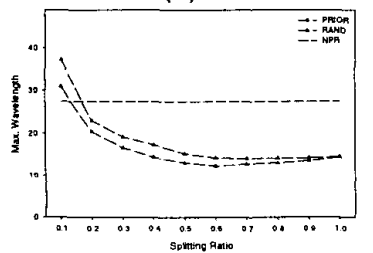

(c)

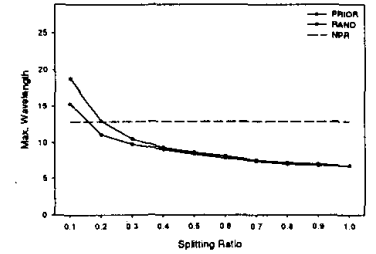

(b)

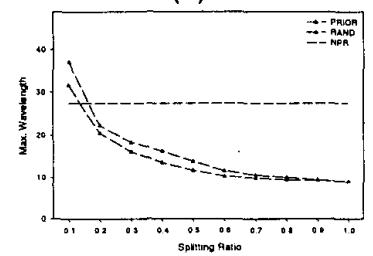

(d)
Figure 8. Maximum number of reserved wavelengths in a link: (a) $D=5$ for SPT, (b) $D=5$ for DDMC, (c) $D=10$ for SPT, and (d) $D=10$ for DDMC

source-to-destination delay because the heuristic for NPR uses the shortest path algorithm. Besides, performance metrics for the MCG tree and for the MIG tree will be the same when the splitting ratio ranges from 0.4 to 0.6 . When the splitting ratio is larger than 0.6 , the implementation cost increases rapidly although the performance metrics are slightly better. Moreover, PRIOR performs better than RAND. That is, if a node has a higher degree in the network, it is necessary to have the multicast capable devices. If the degree of the node is 2 , it is optional to be an MC node. Therefore, all-optical multicasting can be executed efficiently with AOMH.

\section{Conclusions}

We have proposed an all-optical multicasting heuristic (AOMH) method for WDM networks. According to AOMH, the determined multicast tree is a real all-optical multicast tree for WDM networks. In addition, AOMH results in good performance when the number of $\mathrm{MC}$ nodes is larger than $20 \%$ of the number of nodes in the network. Besides, if a node has a higher degree in the network, it is necessary to have multicast capable devices.

Throughout the discussion of this paper, the MCG trees are demonstrated with the source-based and share-based heuristics. In addition, the MIG trees are constructed with the shortest path heuristics. However, AOMH cannot directly guarantee the delay-constrained applications from the source to each destination. In fact, this may be guaranteed with two possible ways. Fist, the MIG lightpaths can be constructed with the delay-constrained heuristics according to the delay of the root specified by the MCG tree. Second the delay-constrained MCG tree can be developed according to the delays of leaves specified by the MIG trees. Therefore, AOMH can support all kinds of multicast applications on WDM networks.

\section{References}

[1] B. Mukherjee, Optical Communication Networks, McGraw Hill, 1997.

[2] J. S. Turner, "New Directions in Communications (or Which Way to the Information Age?)," IEEE Commun. Mag., vol. 24, no. 10, pp. 8-15, Oct. 1986.

[3] E. W. Dijkstra, "A Note on Two Problems in Connection with Graphs," Numerische Mathemtick, vol. 1, pp. 269-271, 1959.

[4] J. Moy, "Multicast Routing extensions for OSPF," Comm. $A C M$, vol. 37, no. 8, pp. 61-66, Aug. 1994.

[5] T. Pusateri, "Distance Vector Routing Protocol," IETF Draft, draft-ietf-idmr-dvmrp-v3-03.txt, Sep. 1996.

[6] K. Carlberg and J. Crowcroft, "Building Shared Trees Using a One-to-Many Joining Mechanism," ACM Computer Communication Review, pp.5-11, Jan. 1997.

[7] L. H. Sahasrabuddhe and B. Mukherjee, "Light-Trees: Optical Multicasting for Improved Performance in Wavelength-Routed Networks," IEEE Commun. Mag., vol. 37, no. 2 , pp. 67-73, Feb. 1999.

[8] R. K. Pankaj, "Wavelength Requirements for Multicasting in All-Optical Networks," IEEE Trans. Networking, vol. 7, no. 3, pp.414-424, June. 1999.

[9] X. Zhang, J. Wei and C. Qiao, "On Fundamental Issues in IP over WDM Multicast," IC3N'99, pp. 84-90, Oct. 1999.

[10] X. Zhang, J. Wei and C. Qiao, "Constrained Multicast Routing in WDM Networks with Sparse Light Splitting," IEEE INFOCOM'2000, pp. 1781-1790, Mar. 2000.

[11] V. Kumar, "MBone: Interactive Multimedia on the Internet," IN: New Riders, 1996.

[12] K. -C. Lee and V. O. K. Li, "A Wavelength Convertible Optical Network," IEEE/OSA J. Lightwave Tech., vol. 11, pp. 962-970, May/June 1993

[13] C. Chen and S. Banerjee, "A New Model for Optimal Routing and Wavelength Assignment in Wavelength Division Multiplexed Optical Networks," INFOCOM'96, pp. 164-171, Mar. 1996.

[14] K. Bala, Routing in Linear Lightwave Networks, doctorial dissertation, Columbia Univ., Dept. Electrical Engineering, N.Y., USA, 1993.

[15] C. E. Leiserson, "Fat-Tree, Universal Network for Hardware-Efficient Supercomputing," IEEE Trans. Computer, vol. C-34, no. 10, pp. 892-901, Oct. 1985.

[16] B. M. Waxman, "Routing of Multipoint Connections," IEEE J. Select. Areas Commun., vol. 6, no. 9, pp. 1617-1622, Dec. 1988.

[17] J. M. S. Doar, Multicasting in The Asynchronous Transfer Mode Environment, doctorial dissertation, Tech. Report No. 298, Computer Laboratory, Univ. Cambridge, 1993. 